\title{
Exploring adherence and attitude towards the Mediterranean diet in a Spanish population
}

\author{
A POSTA* \\ Department of Dietetics, Saint Roch Hospital, Budapest, Hungary
}

(Received: July 16, 2019; revised manuscript received: October 20, 2019; accepted: October 25, 2019)

\begin{abstract}
Purpose: The traditional Mediterranean diet (MD) is recognized worldwide as one of the healthiest existing dietary patterns. However, over the past few decades, a significant deviance from this diet has been observed in a number of Mediterranean countries. The purpose of this study was to assess the Spanish population's degree of deviance from the MD and to explore the possible causes. Materials and methods: A survey was completed by a total of 126 Spanish citizens over the age of 18 years. Dietary and lifestyle habits were assessed using MEDLIFE, a validated index, which was complemented with a questionnaire developed for this study, as well as a 24-hour recall. Results: The mean MEDLIFE score of $15 \pm 3.07$ revealed the average adherence to the MD, while the maximum score was 28 . This study showed no significant positive correlation between MEDLIFE scores and age. The mean ratio of macronutrient intake was $20 \% \mathrm{E}$ protein, $47 \% \mathrm{E}$ carbohydrates, and $33 \% \mathrm{E}$ fat. Conclusions: The results of the study indicate a deviation from several components of the MD. Low MD adherence could be associated with insufficient knowledge regarding the exact composition and health effects of the MD.
\end{abstract}

Keywords: Mediterranean diet, adherence, MEDLIFE index, 24h recall

\section{INTRODUCTION}

Inclusion of the Mediterranean diet (MD) on the prestigious UNESCO World Heritage list (2010) is an inarguable recognition of its cultural value and its role in preserving health. It is associated with values such as frugality and diversity, and, among countless other advantages, is strongly associated with low cardiovascular morbidity [1] and low incidence of cancer. It is recognized worldwide as one of the healthiest, most balanced eating patterns $[2,3]$.

The exact components of the MD may vary slightly depending on the specific country and region; however, the pyramid published in 2011 by the Spanish Mediterranean Diet Foundation offers a graphic overview of its main principles and components, including lifestyle elements such as physical activity and social interaction, both of which play a vital part in the Mediterranean way of life. As shown in Figure 1, the MD is primarily a plant-based diet, characterized by high consumption of vegetables, legumes, fruits, and grains. This is paired with a moderate consumption of dairy products, white meat, and seafood, whereas consumption of red meat, processed meat, and sweets is low. Other typical elements of the MD include olives and olive oil, as well as red wine, consumed in moderation.

Unfortunately, due to an accelerated pace of life over the past few decades, a significant shift from these traditional dietary habits has been observed in a number of Mediterranean countries. The vast variety of convenience foods available makes a more comfortable way of life possible, inclining many to choose store-bought products or fast-food meals over spending time in the kitchen. Thus, while the MD is propagated by healthcare professionals all over the world, the Mediterranean populations themselves may gradually be deviating from this healthy lifestyle.

Several studies have recently explored this topic from different aspects, examining how sociodemographic and lifestyle factors affect nutritional habits. For instance, a higher degree of physical activity has more often been associated with a higher adherence to the MD [4], whereas a sedentary lifestyle (high level of screen time) is generally paired with significantly less optimal nutritional habits [5]. The female gender, a more optimal body mass, and a higher level of nutritional knowledge also show a positive correlation with $\mathrm{MD}$ adherence [6].

Several studies have focused on assessing the lifestyle of the younger generation, as they represent an important target group for nutritional education and public health campaigns. The results consistently indicate a lower MD adherence among the younger age group compared to their elder peers. In 2013, nutritional habits of Spanish university students were evaluated using $24 \mathrm{~h}$ recalls. The mean intake of fatcarbohydrate-protein macronutrients was $40 \%-40 \%-20 \%$ of total energy, respectively. Although the relatively high degree of fat consumption observed correlates with the fact that the $\mathrm{MD}$ is traditionally rich in polyunsaturated fatty acids, in this

* Corresponding address: Adrienn Posta; Department of Dietetics, Saint Roch Hospital, Szabina út 17, Budapest H-1221, Hungary; Phone: +36 2053587 19; E-mail: postaadri@gmail.com

This is an open-access article distributed under the terms of the Creative Commons Attribution-NonCommercial 4.0 International License, which permits unrestricted use, distribution, and reproduction in any medium for non-commercial purposes, provided the original author and source are credited, a link to the CC License is provided, and changes - if any - are indicated. 

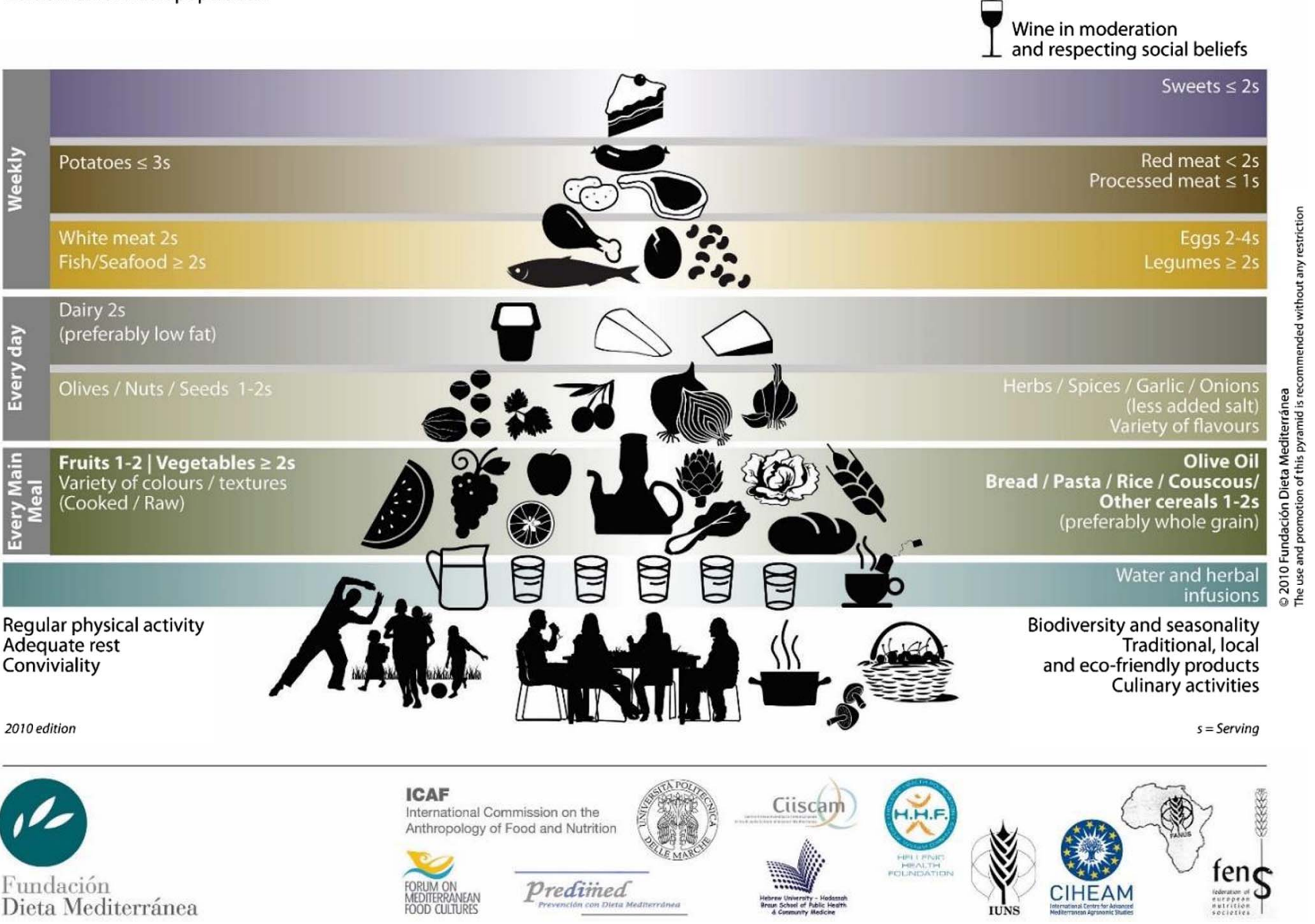

Figure 1. The Mediterranean diet pyramid (source: Fundación Dieta Mediterránea, https://dietamediterranea.com/en/nutrition/)

case, the ratio of the particular fatty acids was far from optimal; furthermore, cholesterol intake was also high [7].

The study "DIMERICA," conducted in 2016, aimed to assess the state of health and nutritional habits of the Spanish population, dividing the country into five geographical areas. Incidence of hypertension, dyslipidaemia, and diabetes was recorded. The results affirmed previous evidence that a lower MD adherence is a strong predictor of cardiovascular risk factors such as hypertension and high blood cholesterol levels.

A new finding of this study was that significant differences were observed between different areas of the country in terms of nutritional habits. Interestingly, the eastern coast of Spain (including Barcelona and its surroundings) showed the poorest nutritional habits, with vegetable, fruit, and fish consumption being the most suboptimal. Overall, a low MD adherence was observed, especially among the young generation. A positive finding of the DIMERICA study was that the use of olive oil seems to still be characteristic of the Spanish population, with $93 \%$ of the participants claiming to consume olive oil daily, and $90 \%$ using it as the primary type of oil for cooking [8].

\section{Methods used in the aforementioned surveys}

Several different indexes have been developed for the assessment of MD adherence over the years, for example,
MD Score, mMDS, and MEDAS. These are all semiquantitative food-frequency questionnaire-based indexes, which generally examine roughly $8-14$ nutritional elements, including consumption of fruit and vegetables, wine, olive oil, grains, poultry, red meat, nuts, and dairy products. One point is assigned when consumption of the given element reaches the quantity defined as the minimum criterion. Based on the total score, MD adherence can be categorized as optimal, average, poor, etc.

In 2014, Sotos-Prieto and her team developed the MEDLIFE index, a questionnaire consisting of 28 elements, which offered a more comprehensive assessment of nutritional and lifestyle habits compared to previous methods. Thus, in this study, the MEDLIFE index is used to assess MD adherence [9].

\section{Objectives}

The objectives of this study were to assess the degree of adherence to the MD and to explore the possible causes of its potential decrease in prevalence among Spanish citizens. Our hypothesis was that the younger generation would be more influenced by modern lifestyle factors that might lead to suboptimal nutritional habits, so we focused on the potential differences among three generations. We explored the subject from a wide variety of aspects, 
including the participants' knowledge regarding composition and health benefits of the MD, as well as their subjective opinions concerning its current prevalence in Spain.

\section{MATERIALS AND METHODS}

\section{Participants}

The 126 participants in this study were readily accessible Spanish citizens over the age of 18 years. They participated voluntarily. The original concept was to compare three generations distant in age; however, the number of older subjects who enrolled was significantly lower than anticipated, so we eventually opted for the age categorization used in several European studies (e.g., European Health Interview Survey). Table 1 demonstrates the gender and age distribution of the participants.

\section{Methods}

A bespoke questionnaire was developed for this study, made available to the participants through Google Forms, as well as on paper. All data were analysed in Microsoft Excel, using the Descriptive Statistics Tool (mean, standard deviation, as well as minimum and maximum values were calculated). The questionnaire consisted of the following elements:

Participant characteristics:

- Sociodemographic variables such as gender, age, level of education, and profession.

- Self-reported anthropometric data [height and weight from which body mass index (BMI) was derived].

- Factors related to state of health: known current diseases, special diets, smoking habits, alcohol consumption, and level of physical activity.

- 28 elements of the validated MEDLIFE index to assess MD adherence. These are shown in Table 2, along with the scoring criteria defined by the authors [9].

- Questions exploring participants' knowledge of and attitude towards the MD, including knowledge of its composition and characteristics, subjective perception of the population's adherence to the MD, thoughts on possible reasons for its decrease in prevalence, and thoughts on its known health benefits. These were all open-ended questions not to influence the participants with preformulated answers.

- Finally, participants were asked to register a day's worth of food consumption in the form of a $24 \mathrm{~h}$ recall, the data from which was evaluated using the software NutriComp Étrend Sport 3.0 (NutriComp L.P., Budapest, Hungary).

It is noteworthy that the questionnaire also included questions exploring the degree of nutritional consciousness, such as questions about grocery shopping habits, number of meals consumed per day, dietary supplement use, calculation of calorie and/or nutrient intake, eating out (frequency and types of restaurants visited), and main sources of information regarding nutrition. However, the present article does not discuss these aspects in detail.
Table 1. Participants' age and gender distribution $(N=126)$

\begin{tabular}{lccccc}
\hline \multirow{2}{*}{$\begin{array}{l}\text { Age groups } \\
\text { (years) }\end{array}$} & \multicolumn{2}{c}{ Female $(n=81)$} & & \multicolumn{2}{c}{ Male $(n=45)$} \\
\cline { 2 - 3 } \cline { 6 - 7 } & Frequency & $\%$ & & Frequency & $\%$ \\
\hline $18-34(n=60)$ & 46 & 36.51 & & 14 & 11.11 \\
$35-64(n=58)$ & 32 & 25.40 & & 26 & 20.63 \\
$65+(n=8)$ & 3 & 2.38 & & 5 & 3.97 \\
\hline
\end{tabular}

\section{RESULTS}

\section{MEDLIFE scores}

Figure 2 illustrates the mean MEDLIFE scores calculated according to the different variables investigated (gender, age, BMI, level of education, profession, and smoking). The average MEDLIFE score was $15 \pm 3.07$, with no significant difference with respect to any variable.

Adherence was also evaluated separately for each MEDLIFE element. Figure 3 illustrates the percentage of participants who adhered to the given elements in the required degree to achieve the score. Considerable differences can be observed between adherence to the specific elements. According to the MEDLIFE index, the most adhered-to element was an adequate consumption of potatoes, whereas the least adhered-to was wine consumption.

\section{Participants' knowledge of and attitude towards the MD}

Figure 4 summarizes the main characteristics of the MD according to the participants. In this open-ended question, subjects were asked to define the MD in terms of its main components and/or characteristics (participants could mention any number of elements). Frequent consumption of fruits and vegetables was the characteristic most often mentioned by participants (69\% and 67\%, respectively). Other characteristics were mentioned with significantly lower frequency. For instance, regular consumption of fish and olive oil was mentioned by only $43 \%$ and $37 \%$ of subjects, respectively. Eighteen percent of participants emphasized that the MD is a balanced and varied diet, whereas $17 \%$ mentioned attention to fresh ingredients.

Questions exploring participants' perception of the population's deviation from the MD were also included in the questionnaire. The question, "Do you think the Spanish population adheres to the MD to the same degree as 30 years ago?" was posed, to which $41 \%$ of the participants answered "No, less," a third answered "Yes, more or less," and $24 \%$ answered "No, much less." A negligible number of people answered "Yes, absolutely."

Participants who thought MD adherence had declined over the past few decades (i.e., those who chose the answers "No, less" or "No, much less") were asked to elaborate in their own words on exactly what this change consisted of and/or the possible reasons for this deviation. The most recurrent reason was lack of time, with one participant phrasing it in the following way: "Today's work hours aren't compatible with the Mediterranean diet." Twelve percent of participants mentioned that an accelerated lifestyle is leading to significant changes in eating habits. 
Table 2. MEDLIFE index

\begin{tabular}{|c|c|c|c|}
\hline Component & Criteria for 1 point & Component & Criteria for 1 point \\
\hline Sweets & $\leq 2$ servings/week & Grains & 3-6 servings/day \\
\hline Red meat & $<2$ servings/week & Water or infusions & $\begin{array}{l}6-8 \text { glasses/day or } \geq 3 \\
\text { portions/week }\end{array}$ \\
\hline Processed meat & $\leq 1$ serving/week & Wine & $1-2$ servings/day \\
\hline Eggs & 2-4 servings/week & Do you limit salt in meals? & Yes \\
\hline Legumes & $\geq 2$ servings/week & Preference for whole grain products & Yes \\
\hline Poultry & 2 servings/week & Salty snacks & $\leq 2$ servings/week \\
\hline Fish/seafood & $\geq 2$ servings/week & Do you limit nibbling between meals? & Yes \\
\hline Potatoes & $\geq 3$ servings/week & $\begin{array}{l}\text { Do you limit sugar in beverages } \\
\text { (including sugar-sweetened beverages) }\end{array}$ & Yes \\
\hline Low-fat dairy products & 2 servings/day & Physical activity & $\begin{array}{l}>150 \mathrm{~min} / \text { week or } \\
30 \mathrm{~min} / \text { day }\end{array}$ \\
\hline Nuts and olives & $1-2$ servings/day & Siesta/nap during weekends & Yes \\
\hline Herbs and spices & $\geq 1$ serving/day & Hours of sleep during weekdays & $6-8 \mathrm{hr} /$ day \\
\hline Fruit & 3-6 servings/day & Television during weekdays & $<1 \mathrm{hr} /$ day \\
\hline Vegetables & $\geq 2$ servings/day & Socializing with friends during weekends & $\geq 2 \mathrm{hr} /$ weekend \\
\hline Olive oil & $\geq 3$ servings/day & Collective sports & $\geq 2 \mathrm{hr} /$ week \\
\hline
\end{tabular}

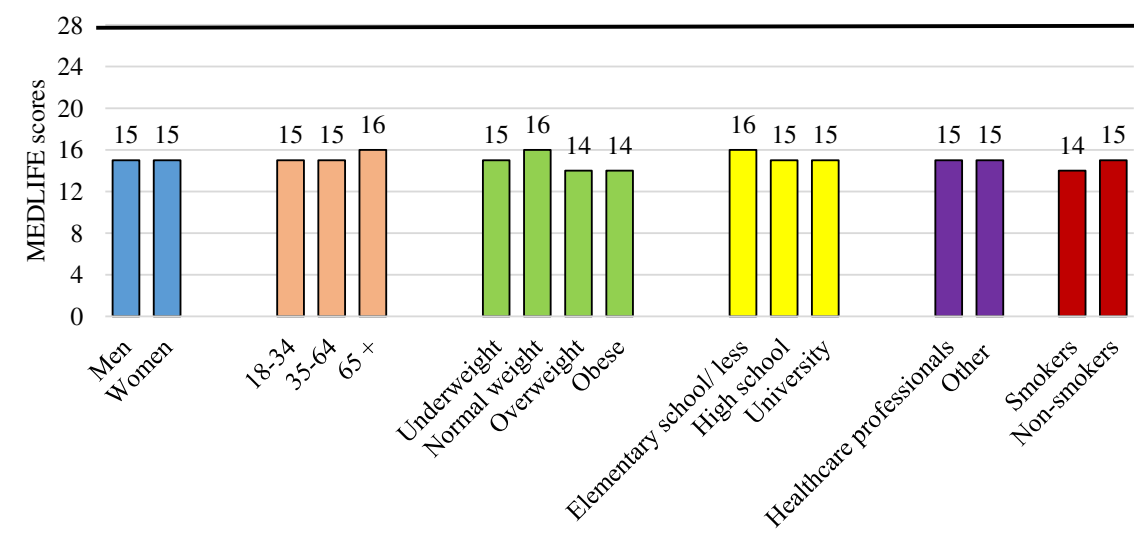

Figure 2. MEDLIFE index scores based on gender, age group, BMI, highest level of education, profession, and smoking categories $(N=126$; horizontal black line indicates the reference value)

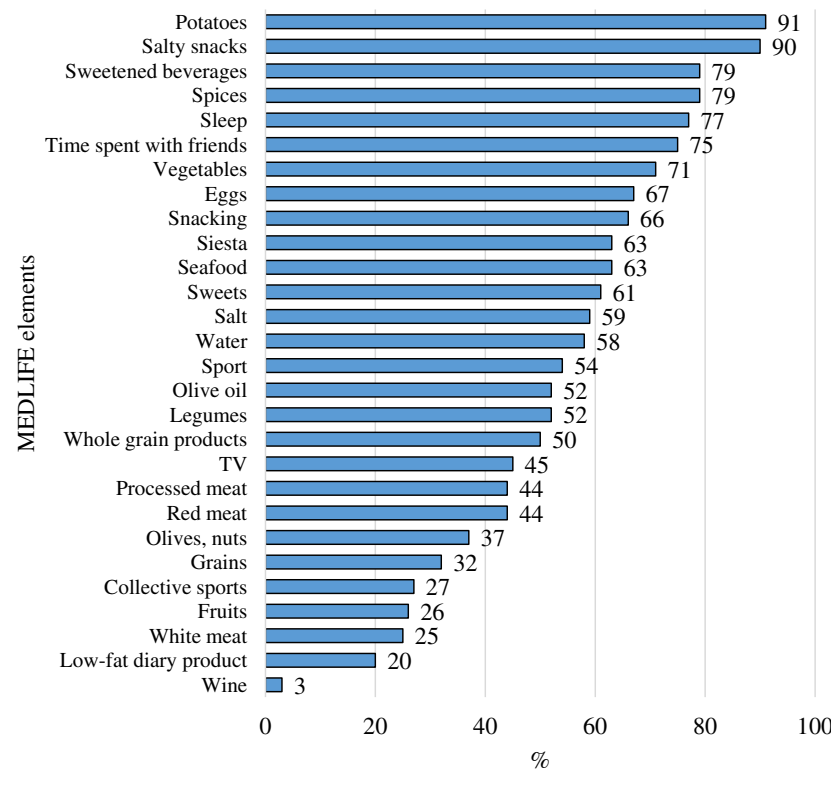

Figure 3. Percentage adherence to the specific MEDLIFE elements

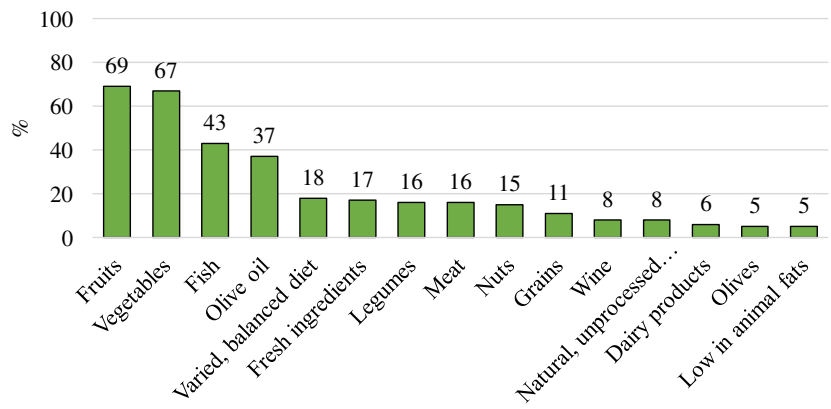

Figure 4. Main components/characteristics of the MD according to participants $(N=126)$

A third of the participants answered that the change consisted of fast food, and another third answered that it consisted of convenience and processed foods. Nine percent of participants answered that international cuisines are also "to blame" (several participants mentioned that these "often offer cheaper meals"). Seven percent of participants 
answered that the reason was globalization and $4 \%$ answered that the reason was eating out. Other intriguing answers mentioned by a few participants include: "While the science of dietetics is growing, our nutritional habits are getting worse and worse because of the fast food." One participant mentioned the role of food advertisements, saying "they aggressively try to persuade us to buy products we don't need." Another interesting answer was "We are inclined to snack, rather than eat." Several answers defined the specific ways in which this change manifests itself: lower consumption of fruits, vegetables, and legumes, paired with a higher consumption of deep-fried foods, red meat, store-bought sweets, and other carbohydrate-dense foods. Several participants mentioned that consumption of beer is much more typical today than wine. A few people answered that the seasonality of fruits and vegetables is not really considered anymore, since they are now available across several seasons.

When asked about the health benefits of the MD, the majority (77\%) of participants answered that the MD is a balanced eating pattern. Seventy-three percent of participants answered that it had a positive effect on cardiovascular health. Half of the participants knew of its role in preserving a healthy body weight, whereas only $39 \%$ of participants agreed that it had beneficial effects with regard to carbohydrate metabolism disorders such as diabetes. Only a similarly low number of people believed it was beneficial in preventing cancer. A negligible number of people believed that the MD had no health benefits at all.

\section{$24 h$ recall}

The mean energy intake calculated from the $24 \mathrm{~h}$ recalls was $1,487.2 \pm 456 \mathrm{kcal}$. The average ratio of macronutrient intake was $20 \pm 5.31 \mathrm{E} \%$ protein, $47 \pm 11.06 \mathrm{E} \%$ carbohydrates, and $33 \pm 9.37 \mathrm{E} \%$ fat. The relatively high ratio of fat intake - typical of the traditional MD - was paired, however, with an inadequate ratio of the different fatty acids. For instance, the omega-6 to omega-3 fatty acid ratio of approximately 10:1 suggests that omega- 6 fatty acids consumption is at least threefold higher than optimal. The inflammatory effects of this can significantly elevate cardiovascular risk.

\section{DISCUSSION}

The mean MEDLIFE score of $15 \pm 3.07$ revealed the average adherence to the MD, while the maximum score was 28 . Contrary to our hypothesis, there was no significant difference in MEDLIFE scores correlated with different age groups or any other variable examined. However, this could be a consequence of the small number of participants, especially those representing the older generation. The small number of participants was the study's biggest limitation.

By evaluating the answers to questions concerning participants' subjective thoughts and opinions about the MD and its prevalence, several conclusions can be drawn. Based on these results, a lack of knowledge regarding the exact composition and various health benefits of the MD could play a role in the decreasing adherence. Therefore, it is crucial that Spanish citizens learn from a young age, not only the practice of, but also the theory behind the components of the MD and its health benefits, especially since the majority of participants derive nutrition-related information from questionable sources on the Internet, rather than from healthcare professionals.

A significant number of the participants were aware of the shift taking place from their traditional diet, and they have fixed theories regarding the possible reasons (they primarily listed lack of time and the spreading of fast food). That said, the question arises whether these are in fact the reasons, and what the most efficient way to influence the situation might be.

\section{CONCLUSIONS}

The results of this study indicate a deviation from several components of the traditional MD among Spanish subjects. This supports previous findings on this topic. While the MEDLIFE index offered a strong basis when developing the questionnaire for this study, other factors evaluated (that did not form part of a previously validated survey) also proved to be informative. An added value of this study compared to those cited in the introduction is that it also offered an insight into the Spanish participants' subjective opinions about the topic.

Taking several health benefits of the MD into account, it is important to multidimensionally monitor the dietary and lifestyle habits of the Mediterranean populations, where the appropriate geographical and climatic conditions are given. Not only does a decreasing MD adherence have a negative impact on the population's state of health, but it could also result in the MD's good reputation and authenticity being lost, which could lead to its decrease in prevalence all over the world.

Recent trials offer conflicting conclusions regarding the relationship between dietary cholesterol and saturated fatty acid intake and cardiovascular morbidity, in light of which several aspects of the MD should be reevaluated $[10,11]$.

Acknowledgements: The author would like to thank Dr. Lichthammer Adrienn for her guidance and help throughout this research.

Ethical approval: All procedures were in accordance with the ethical standards of the institutional and/or national research committee and with the 1964 Helsinki Declaration and its later amendments or comparable ethical standards. Participants received written information about the study.

Conflicts of Interest/Funding: The author declares no conflict of interest and no financial support was received for this study.

\section{REFERENCES}

1. Widmer RJ, Flammer AJ, Lerman LO, Lerman A. The Mediterranean diet, its components, and cardiovascular disease. Am J Med. 2015;128(3):229-38. 
2. Schwingshackl L, Hoffmann G. Adherence to Mediterranean diet and risk of cancer: an updated systematic review and meta-analysis of observational studies. Cancer Med. 2015;4(12):1933-47.

3. Di Daniele N, Noce A, Vidiri MF, et al. Impact of Mediterranean diet on metabolic syndrome, cancer and longevity. Oncotarget. 2017;8(5):8947-79.

4. Fauquet J, Sofi F, López-Gimerá G, et al. Mediterranean diet adherence among Catalonian adolescents: socioeconomic and lifestyle factors. Nurt Hops. 2016;33(6): 1283-90.

5. Arriscado D, Muros JJ, Zabala M, Dalmau JM. Factors associated with low adherence to a Mediterranean diet in healthy children in Northern Spain. Appetite. 2014;80: 28-34.

6. Patino-Alonso MC, Recio-Rodríguez JI, Belio JFM, et al. Factors associated with adherence to the Mediterranean diet in the adult population. J Acad Nutr Diet. 2014;114(4): 583-9.
7. García-Meseguer MJ, Burriel FC, Garcíia CV, Serrani-Urrea R. Adherence to Mediterranean diet in a Spanish university population. Appetite. 2014;78:156-64.

8. Abellán Alemán J, Zafrilla Rentero MP, Montoro-García S, et al. Adherence to the "Mediterranean Diet" in Spain and its relationship with cardiovascular risk (DIMERICA Study). Nutrients. 2016;8(11):680.

9. Sotos-Prieto M, Moreno-Franco B, Ordovás JM, León M, Casasnovas JA, Peñalvo JL. Design and development of an instrument to measure overall lifestyle habits for epidemiological research: the Mediterranean Lifestyle (MEDLIFE) Index. Public Health Nurt. 2015;18(6):959-67.

10. Dehghan M, Menta A, Zhang X, et al. Associations of fats and carbohydrate intake with cardiovascular disease and mortality in 18 countries from five continents (PURE): a prospective cohort study. Lancet. 2017;390(10107):2050-62.

11. Astrup A, Bertram HC, Bonjour JP, et al. WHO draft guidelines on dietary saturated and trans fatty acids: time for a new approach? BMJ 2019;366:15683. 